TRANSACTIONS OF THE

AMERICAN MATHEMATICAL SOCIETY

Volume 362, Number 4, April 2010, Pages 1981-200

S 0002-9947(09)04746-1

Article electronically published on November 16, 2009

\title{
SINGULARLY PERTURBED NONLINEAR DIRICHLET PROBLEMS WITH A GENERAL NONLINEARITY
}

\author{
JAEYOUNG BYEON
}

\begin{abstract}
Let $\Omega$ be a bounded domain in $\mathbf{R}^{n}, n \geq 3$, with a boundary $\partial \Omega \in C^{2}$. We consider the following singularly perturbed nonlinear elliptic problem on $\Omega$ :

$$
\varepsilon^{2} \Delta u-u+f(u)=0, \quad u>0 \text { on } \Omega, \quad u=0 \text { on } \partial \Omega,
$$

where the nonlinearity $f$ is of subcritical growth. Under rather strong conditions on $f$, it has been known that for small $\varepsilon>0$, there exists a mountain pass solution $u_{\varepsilon}$ of above problem which exhibits a spike layer near a maximum point of the distance function $d$ from $\partial \Omega$ as $\varepsilon \rightarrow 0$. In this paper, we construct a solution $u_{\varepsilon}$ of above problem which exhibits a spike layer near a maximum point of the distance function under certain conditions on $f$, which we believe to be almost optimal.
\end{abstract}

\section{INTRODUCTION}

Let $\Omega$ be a bounded domain in $\mathbf{R}^{n}, n \geq 3$, with a $C^{2}$ boundary $\partial \Omega$. We are interested in the following singularly perturbed nonlinear elliptic problem on $\Omega$ :

$$
\varepsilon^{2} \Delta u-u+f(u)=0, \quad u>0 \text { on } \Omega, \quad u=0 \text { on } \partial \Omega,
$$

where a typical form of $f(u)$ is $|u|^{p-1} u, p \in(1,(n+2) /(n-2))$. The most basic solution for (1) is a mountain pass solution. For the existence of a mountain pass solution for (11), the following conditions have been basically assumed in the literature. For a continuous function $f: \mathbf{R} \rightarrow \mathbf{R}$,

(f1) $f(t)=0$ for $t \leq 0$ and $\lim _{t \rightarrow 0} f(t) / t=0$;

(f2) there exists $p \in\left(1, \frac{n+2}{n-2}\right)$ such that $\limsup _{t \rightarrow \infty} f(t) / t^{p}<\infty$;

(f3-1) there exist $\mu>2$ and $t_{0}>0$ such that $\mu \int_{0}^{t} f(s) d s<f(t) t$ for $t>t_{0}$.

Furthermore, if we assume additional strong conditions $f \in C^{1}$, (f1), (f2) and

(f3-2) there exist $\mu>2$ such that $\mu \int_{0}^{t} f(s) d s<f(t) t$ for $t>0$,

for small $\varepsilon>0$, there exists a unique maximum point $x_{\varepsilon} \in \Omega$ of a mountain pass solution $u_{\varepsilon}$ such that a transformed function $v_{\varepsilon}(x) \equiv u_{\varepsilon}\left(\varepsilon\left(x-x_{\varepsilon}\right)\right)$ converges uniformly to a radially symmetric least energy solution of the following limiting problem:

$$
\Delta u-u+f(u)=0, \quad u>0 \text { in } \mathbf{R}^{n} \text { and } \lim _{|x| \rightarrow \infty} u(x)=0 .
$$

Received by the editors October 4, 2006 and, in revised form, December 12, 2007.

2000 Mathematics Subject Classification. Primary 35J65, 35J20.

This work was supported by the Korea Research Foundation Grant funded by the Korean Government (MOEHRD, Basic Research Promotion Fund) (KRF-2007-313-C00047).

(C)2009 American Mathematical Society Reverts to public domain 28 years from publication 
Thus, $u_{\varepsilon}$ develops a spike layer as $\varepsilon \rightarrow 0$. In their striking paper [23, $\mathrm{Ni}$ and Wei proved that the point $x_{\varepsilon}$ of the spike layer converges to the most centered points of $\Omega$, that is, the maximum points of the distance function $d$ from $\partial \Omega$ as $\varepsilon \rightarrow 0$. For the proof, they required the following additional conditions:

(f4) $f(t) / t$ is nondecreasing on $(0, \infty)$;

(f5) there exists a unique radially symmetric solution $U \in H^{1,2}\left(\mathbf{R}^{n}\right)$ for $\Delta u-$ $u+f(u)=0, u>0$, in $\mathbf{R}^{n}$ such that if $\Delta V-V+f^{\prime}(U) V=0$ and $V \in$ $H^{1,2}\left(\mathbf{R}^{n}\right)$, then $V=\sum_{i=1}^{n} a_{i} \frac{\partial U}{\partial x_{i}}$ for some $a_{1}, \cdots, a_{n} \in \mathbf{R}$.

(See [13, 21], 24] and 27] for more works on higher energy solutions.) Furthermore, del Pino and Felmer [12] showed the asymptotic behavior of the spike layer by a more simple approach without the nondegeneracy condition (f5). Finally, the author 4 ] proved the asymptotic behavior without the additional conditions (f4) and (f5) for $n \geq 3$.

There have also been similar works [10], [1], 15], 17], 22] for the problem (11) when the nonlinearity $f$ is typically of the form $f(u)=u-u(u-a)(u-1)$, $0<a<\frac{1}{2}$. A culminating result in this direction was obtained by $\mathrm{Ni}$, Takagi and Wei [22]. The result says that if $\Omega$ satisfies a geometric condition $(\mathcal{C})$, the same phenomena with the case $f$ satisfying (f1), (f2) and (f3) occur for a mountain pass solution $u_{\varepsilon}$. Convex sets and annuli satisfy condition $(\mathcal{C})$, but not domains with two or more holes (see 22] for the definition of condition $(\mathcal{C})$ ). Their assumptions for $f(t) \equiv t+g(t)$ are the following:

(g1) $g \in C^{1+\sigma}$ for some $\sigma \in(0,1)$ and $g(0)=0, g^{\prime}(0)<0$;

(g2) $g$ has two positive zero $z_{1}$ and $z_{2}$, such that $z_{1}<z_{2}$ and $g$ has no other positive zeros;

(g3) $\int_{0}^{z_{2}} g(s) d s>0$

(g4) the function $t \rightarrow f(t) /\left(t-t_{0}\right)$ is decreasing in the interval $\left(t_{0}, z_{2}\right)$, where $t_{0}$ is the unique number in $\left(z_{1}, z_{2}\right)$ such that $\int_{0}^{t_{0}} f(s) d s=0$.

In two or three dimensional cases, Dancer [11] proved the same phenomena without the geometric condition $(\mathcal{C})$.

On the other hand, Berestycki and Lions had showed in the classical paper [9] that equation (2) has a radially symmetric least energy solution $U$ satisfying, for some $C, \delta>0$ and any $|\alpha| \leq 2$,

$$
\left|D^{\alpha} U(x)\right| \leq C \exp (-\delta|x|), \quad x \in \mathbf{R}^{n},
$$

under conditions (f1), (f2) and the following condition:

(f3) there exists $T>0$ satisfying $F(T) \equiv \int_{0}^{T} f(t) d t>T^{2} / 2$.

Moreover, they showed that for any solution $U \in H^{1}$ of (2), the following Pohozaev identity holds:

$$
\frac{n-2}{2} \int_{\mathbf{R}^{n}}|\nabla U|^{2} d x+\frac{n}{2} \int_{\mathbf{R}^{n}} U^{2} d x-n \int_{\mathbf{R}^{n}} F(U) d x=0 .
$$

We believe that conditions (f1), (f2) and (f3) are almost optimal for an existence of solutions for (2). In fact, it follows from the Pohozaev identity (4) that condition (f3) is necessary and that for $f(u)=u^{p}, p \geq \frac{n+2}{n-2}$, there exist no solutions of (2) in $H^{1,2}\left(\mathbf{R}^{n}\right)$. When $f$ satisfies (g1), (g2), (g3) and (g4), the mountain pass solution considered in [10, 15], 17] and 22] has its range in $\left(0, z_{2}\right)$. Thus, redefining $g(t)=0$ for $t \geq z_{2}$, we see that conditions (g1), (g2), (g3) and (g4) are stronger than 
conditions (f1), (f2) and (f3). In [18, Jeanjean and Tanaka noticed that a least energy solution of (2) is a mountain pass solution. Since the mountain pass solution is structurally stable, it seems natural to expect that there exists a corresponding solution of a singularly perturbed problem (11) for small $\varepsilon>0$ whenever a limiting problem (2) has a least energy solution. In this paper we will prove that indeed, for $n \geq 3$, there exists a perturbed solution and its maximum point converges to a most centered point of $\Omega$ as $\varepsilon \rightarrow 0$. Since we assume only (f1), (f2), (f3) in this paper, our approach unifies both results under conditions (f1), (f2), (f3-2) and under (g1), (g2), (g3), (g4). Furthermore we do not need any geometric condition on domain $\Omega$ and any additional smoothness of $f$. Our approach for an existence of a solution for small $\varepsilon>0$ is basically variational, but takes advantage of a reduction to a compact set in a finite dimensional reduction method used powerfully when the nondegeneracy condition (f5) is satisfied. This approach was successfully carried out in the study of the standing waves for nonlinear Schrödinger equations [5], 6], [7.

We define $\mathcal{S}$ as the set of a solution $U$ for (21) satisfying

$$
U(0, \cdots, 0,0)=\max _{x \in \mathbf{R}^{n}} U(x) .
$$

Since we assume just that $f$ is continuous, it is not certain whether any solution $u \in \mathcal{S}$ is radially symmetric and monotone decreasing. A result in 9 says that if (f1), (f2) and (f3) are satisfied, there exists a radially symmetric solution $U \in \mathcal{S}$ which is monotone decreasing. Now we state our main results as follows.

Theorem 1.1. We assume that $n \geq 3$ and a function $f \in C(\mathbf{R})$ satisfies conditions (f1), (f2) and (f3). Then, for sufficiently small $\varepsilon>0$, there exists a solution $u_{\varepsilon}$ of (11) and a maximum point $x_{\varepsilon} \in \Omega$ of $u_{\varepsilon}$ such that

(i) for some constant $C, c>0, u_{\varepsilon}(x) \leq C \exp \left(-\frac{c}{\varepsilon} \operatorname{dist}\left(x, x_{\varepsilon}\right)\right)$,

(ii)

$$
\lim _{\varepsilon \rightarrow 0} \operatorname{dist}\left(x_{\varepsilon}, \partial \Omega\right)=\max _{x \in \Omega} \operatorname{dist}(x, \partial \Omega)
$$

and, for a transformed solution $v_{\varepsilon}(x) \equiv u_{\varepsilon}\left(\varepsilon\left(x-x_{\varepsilon}\right)\right)$, a sequence $\left\{v_{\varepsilon}\right\}_{\varepsilon}$ converges, up to a subsequence, uniformly to a least energy solution $U \in \mathcal{S}$ of (2).

This paper is organized as follows. In section 2, we introduce some notation and prepare some preliminary results for the proofs of the main result. In section 3, we consider the problem (2) when the domain $\Omega$ is a ball. Then, combining the results in sections 2 and 3 , we prove our main theorem, Theorem 1.1 .

\section{Preliminaries}

First, we study the related limiting problem (11). The space $H^{1} \equiv H^{1,2}\left(\mathbf{R}^{n}\right)$ is a completion of $C_{0}^{\infty}\left(\mathbf{R}^{n}\right)$ with respect to the norm

$$
\|u\| \equiv\left(\int_{\mathbf{R}^{n}}|\nabla u|^{2}+u^{2} d x\right)^{1 / 2} .
$$

Since we are interested in positive solutions, we may assume that $f(t)=0$ for $t \leq 0$. We define an energy functional $\Gamma$ on $H^{1}$ by

$$
\Gamma(u)=\frac{1}{2} \int_{\mathbf{R}^{n}}|\nabla u|^{2}+u^{2} d x-\int_{\mathbf{R}^{n}} F(u) d x,
$$

where $F(t)=\int_{0}^{t} f(s) d s$. 
Any critical point of $\Gamma$ in $H^{1}$ is a solution of (2). Moreover, any solution $u \in H^{1}$ (not necessarily positive) of (21) is contained in the following set $\mathcal{P}$ :

$$
\mathcal{P} \equiv\left\{\left.u \in H^{1} \backslash\{0\}\left|\frac{n-2}{2} \int_{\mathbf{R}^{n}}\right| \nabla u\right|^{2} d x+n \int_{\mathbf{R}^{n}} \frac{u^{2}}{2}-F(u) d x=0\right\},
$$

which comes from the Pohozaev identity. The existence of a least energy solution for (2) was proved in [9] when $f$ satisfies (f1), (f2), (f3). It is noticed in [18] that any least energy solution is a mountain pass solution. Here we use a different approach, which is more suitable for our proof of the main result in this paper. We consider the following minimization:

$$
I \equiv \inf _{u \in \mathcal{P}} \Gamma(u) .
$$

Proposition 2.1. We assume that $n \geq 3$ and that (f1), (f2) and (f3) hold. Then, there exists a radially symmetric and monotone decreasing minimizer $U \in \mathcal{P} \cap C^{2}$ of (5) which satisfies equation (2). Any minimizer $U$ of (5) is a least energy solution of (2).

Proof. From (f1) and (f3), we see that $\mathcal{P}$ is not empty. Let $\left\{u_{m}\right\}_{m=1}^{\infty}$ be a minimizing sequence of (5). Let $u_{m}^{*}$ be a radially symmetric rearrangement (Schwartz symmetrization) of $u_{m}$, and let $u_{m}^{*, s}(x) \equiv u_{m}^{*}\left(\frac{x}{s}\right)$. Note that

$$
\begin{gathered}
\int_{\mathbf{R}^{n}}\left|\nabla u_{m}^{*, s}\right|^{2} d x=s^{n-2} \int_{\mathbf{R}^{n}}\left|\nabla u_{m}^{*}\right|^{2} d x \leq s^{n-2} \int_{\mathbf{R}^{n}}\left|\nabla u_{m}\right|^{2} d x, \\
\int_{\mathbf{R}^{n}} \frac{\left(u_{m}^{*, s}\right)^{2}}{2}-F\left(u_{m}^{*, s}\right) d x=s^{n} \int_{\mathbf{R}^{n}} \frac{\left(u_{m}\right)^{2}}{2}-F\left(u_{m}\right) d x .
\end{gathered}
$$

Thus, there exists $s_{m} \in(0,1]$ such that $u_{m}^{*, s_{m}} \in \mathcal{P}$. Then, we see that

$$
\begin{aligned}
\Gamma\left(u_{m}^{*, s_{m}}\right) & =\frac{\left(s_{m}\right)^{n-2}}{2} \int_{\mathbf{R}^{n}}\left|\nabla u_{m}^{*}\right|^{2} d x-\left(s_{m}\right)^{m} \frac{n-2}{2 n} \int_{\mathbf{R}^{n}}\left|\nabla u_{m}\right|^{2} d x \\
& \leq\left(\frac{\left(s_{m}\right)^{n-2}}{2}-\left(s_{m}\right)^{m} \frac{n-2}{2 n}\right) \int_{\mathbf{R}^{n}}\left|\nabla u_{m}\right|^{2} d x \\
& \leq\left(\frac{1}{2}-\frac{n-2}{2 n}\right) \int_{\mathbf{R}^{n}}\left|\nabla u_{m}\right|^{2} d x \\
& =\Gamma\left(u_{m}\right) .
\end{aligned}
$$

Thus, we may assume that $u_{m}$ is radially symmetric and monotone decreasing for each $m=1,2, \cdots$. Since $u_{m}$ is contained in $\mathcal{P}$, it follows that $\Gamma\left(u_{m}\right)=$ $\frac{1}{n} \int_{\mathbf{R}^{n}}\left|\nabla u_{m}\right|^{2} d x$; thus $\left\{\left.\int_{\mathbf{R}^{n}} \nabla u_{m}\right|^{2} d x\right\}_{m}$ is bounded. Moreover, it is easy to see from (f2) and Sobolev imbedding that $\left\{\int_{\mathbf{R}^{n}}\left(u_{m}\right)^{2} d x\right\}_{m}$ is bounded. Thus, taking a subsequence if it is necessary, we may assume that $u_{m}$ converges weakly to some radially symmetric function $U$ in $H^{1}$. We denote $H_{r}^{1} \equiv\left\{u \in H^{1} \mid u(x)=u(|x|)\right\}$. Then, it follows from [19] that an imbedding $H_{r}^{1} \hookrightarrow L^{q}\left(\mathbf{R}^{n}\right)$ is compact for $q \in$ $(2,2 n /(n-2))$. Then, it follows that $\lim _{m \rightarrow \infty} \int_{\mathbf{R}^{n}} F\left(u_{m}\right) d x=\int_{\mathbf{R}^{n}} F(U) d x$ and

$$
\lim _{m \rightarrow \infty} \int_{\mathbf{R}^{n}}\left|\nabla u_{m}\right|^{2}+\left(u_{m}\right)^{2} d x \geq \int_{\mathbf{R}^{n}}|\nabla U|^{2}+U^{2} d x .
$$

Note that

$$
\frac{n-2}{2} \int_{\mathbf{R}^{n}}|\nabla U|^{2} d x+n \int_{\mathbf{R}^{n}} \frac{U^{2}}{2}-F(U) d x \leq 0 .
$$


As before, we see that for $U^{s}(x) \equiv U\left(\frac{x}{s}\right)$, there exists $t \in(0,1]$ satisfying $U^{t} \in \mathcal{P}$. Then, as before, it follows that

$$
\Gamma\left(U^{t}\right) \leq \Gamma(U) \leq \lim _{m \rightarrow \infty} \Gamma\left(u_{m}\right) .
$$

This implies that $U^{t} \in H_{r}^{1}$ is a minimizer of (5). For sake of convenience, let $U \in H_{r}^{1}$ be a minimizer of (5). Then, there exists a Lagrange multiplier $\lambda \in \mathbf{R}$ satisfying

$$
\Delta U-U+f(U)+\lambda((n-2) \Delta U-n U+n f(U))=0 \text { in } \mathbf{R}^{n} .
$$

This implies that

$$
(1+(n-2) \lambda) \Delta U-(1+n \lambda) U+(1+n \lambda) f(U)=0 \quad \text { in } \quad \mathbf{R}^{n} .
$$

From the Pohozaev identity (4), we see that

$$
\frac{(1+(n-2) \lambda)(n-2)}{2} \int_{\mathbf{R}^{n}}|\nabla U|^{2} d x+(1+n \lambda) n \int_{\mathbf{R}^{n}} \frac{U^{2}}{2}-F(U) d x=0 .
$$

Since $U \in \mathcal{P}$, it follows that

$$
\lambda(n-2) \int_{\mathbf{R}^{n}}|\nabla U|^{2} d x=0 .
$$

This implies that $\lambda=0$. Thus the minimizer $U$ satisfies equation (2). Since $U$ is radially symmetric, it follows that $U \in C^{2}$. Since any solution of (2) is contained in $\mathcal{P}$, any minimizer of (5) is a least energy solution of (2). This completes the proof.

Take any $V \in H^{1}$ satisfying $\Gamma(V)<0$. Then, we define a mountain pass level $C(V)$ by

$$
C(V) \equiv \inf _{\gamma \in \Psi} \max _{t \in[0,1]} \Gamma(\gamma(t)),
$$

where $\Psi \equiv\left\{\gamma \in C\left([0,1], H^{1}\right) \mid \gamma(0)=0, \gamma(1)=V\right\}$. Then, we will see that $C(V)$ is independent on $V$ satisfying $\Gamma(V)<0$ via the following result, which is well known when $f(t) / t$ is monotone increasing.

Proposition 2.2. We assume that (f1), (f2) and (f3) hold. Suppose that for $V_{1}, V_{2} \in H^{1}, \Gamma\left(V_{i}\right)<0, i=1,2$. Then, there exists a path $g \in C\left([0,1], H^{1}\right)$ such that $g(0)=V_{1}, g(1)=V_{2}$ and $\Gamma(g(t))<0$ for $t \in[0,1]$.

Proof. We find a function $\phi \in C_{0}^{\infty}\left(\mathbf{R}^{n}\right)$ such that $\phi(x)=0$ for $|x| \geq 2$ and $\phi(x)=1$ for $|x| \leq 1$. For $t>0$, we define $\phi_{t}(x)=\phi(t x)$. Then, there exists $t_{0}>0$ such that $\Gamma\left(s V_{i}+(1-s) \phi_{t} V_{i}\right)<0$ for any $s \in[0,1]$ and $i=1,2$. Thus, we may assume that $\operatorname{supp}\left(V_{1}\right) \cup \operatorname{supp}\left(V_{2}\right) \subset B(0, R)$ for some $R>0$. Define $V_{i}^{t}(x) \equiv V_{i}\left(\frac{x}{t}\right)$. Then, we see that

$$
\Gamma\left(V_{i}^{t}\right)=\frac{t^{n-2}}{2} \int_{\mathbf{R}^{n}}\left|\nabla V_{i}\right|^{2} d x+t^{n} \int_{\mathbf{R}^{n}} \frac{\left(V_{i}\right)^{2}}{2}-F\left(V_{i}\right) d x .
$$

Note that

$$
\left.\frac{d \Gamma\left(V_{i}^{t}\right)}{d t}\right|_{t=1}=n \Gamma\left(V_{i}\right)-\int_{\mathbf{R}^{n}}\left|\nabla V_{i}\right|^{2}<0 .
$$


Thus, we see that $\Gamma\left(V_{i}^{t}\right)$ is strictly decreasing for $t \geq 1$. Moreover, from the facts $\int_{\mathbf{R}^{n}} \frac{\left(V_{i}\right)^{2}}{2}-F\left(V_{i}\right) d x<0$ for each $i=1,2$, it follows that $\lim _{t \rightarrow \infty} \Gamma\left(V_{i}^{t}\right)=-\infty$. Then, we can choose $t_{1}>1$ such that

$$
\Gamma\left(V_{1}^{t_{1}}\right) \leq-1-\max _{\theta \in[0,1]}\left|\Gamma\left(\theta V_{2}\right)\right| .
$$

Similarly, we can choose $t_{2}>1$ such that

$$
\Gamma\left(V_{2}^{t_{2}}\right) \leq-1-\max _{\theta \in[0,1]}\left|\Gamma\left(\theta V_{1}^{t_{1}}\right)\right| .
$$

Thus, there exists a path connecting $V_{1}$ and $V_{1}^{t_{1}}$ on which $\Gamma$ is negative. We define $V_{3}^{s}(x) \equiv V_{1}^{t_{1}}\left(x+\left(2 s R\left(t_{1}+t_{2}\right), 0, \cdots, 0\right)\right), s \in[0,1]$. Then, we see that for $s \in[0,1]$,

$$
\Gamma\left(V_{3}^{s}\right)=\Gamma\left(V_{3}^{0}\right)=\Gamma\left(V_{1}^{t_{1}}\right)<0 .
$$

We note that for $t \in\left[1, t_{2}\right], \operatorname{supp}\left(V_{3}^{1}\right) \cap \operatorname{supp}\left(V_{2}^{t}\right)=\emptyset$. Then, we see that for $\theta \in[0,1]$,

$$
\Gamma\left(V_{3}^{1}+\theta V_{2}\right)=\Gamma\left(V_{3}^{1}\right)+\Gamma\left(\theta V_{2}\right)<0 .
$$

Thus, there exists a path connecting $V_{1}$ and $V_{3}^{1}+V_{2}$ on which $\Gamma$ is negative. Since $\Gamma\left(V_{3}^{1}+V_{2}^{t}\right)=\Gamma\left(V_{3}^{1}\right)+\Gamma\left(V_{2}^{t}\right)$ for $t \in\left[1, t_{2}\right]$, there exists a path connecting $V_{3}^{1}+V_{2}$ and $V_{3}^{1}+V_{2}^{t_{2}}$ on which $\Gamma$ is negative. Note that $\Gamma\left(\theta V_{3}^{1}+V_{2}^{t_{2}}\right)=\Gamma\left(\theta V_{1}^{t_{1}}\right)+\Gamma\left(V_{2}^{t_{2}}\right), \theta \in$ $[0,1]$ and $\Gamma\left(V_{2}^{t}\right)<0, t \in\left[1, t_{2}\right]$. Thus it follows from (8) that there exists a path connecting $V_{3}^{1}+V_{2}^{t_{2}}$ and $V_{2}$ on which $\Gamma$ is negative. This completes the proof.

From Proposition 2.2 above, we see that $C \equiv C(V)$ is independent of $V$ satisfying $\Gamma(V)<0$.

We define $H_{r}^{1} \equiv\left\{u \in H^{1,2}\left(\mathbf{R}^{n}\right) \mid u(x)=u(|x|)\right\}$. Then, we have further the following pathwise connectedness in $H_{r}^{1}$.

Proposition 2.3. Let $U, V$ be radially symmetric functions in $H_{r}^{1}$ such that $\Gamma(U)$, $\Gamma(V)<0$. Then, there exists a path $g \in C\left([0,1], H_{r}^{1}\right)$ such that $g(0)=U, g(1)=V$ and $\Gamma(g(t))<0$ for $t \in[0,1]$.

Proof. We can take $a>0$ such that $\Gamma(u)<0$ if $\|U-u\| \leq a$ or $\|V-u\| \leq a$. Since $C_{0}^{\infty}\left(\mathbf{R}^{n}\right) \cap H_{r}^{1}$ is dense in $H_{r}^{1}$, we can take $U_{1}, V_{1} \in C_{0}^{\infty}\left(\mathbf{R}^{n}\right) \cap H_{r}^{1}$ such that $\left\|U-U_{1}\right\| \leq a$ and $\left\|V-V_{1}\right\| \leq a$. Thus, it suffices to find a path $g \in C\left([0,1], H_{r}^{1}\right)$ such that $g(0)=U_{1}, g(1)=V_{1}$ and $\Gamma(g(t))<0$ for $t \in[0,1]$. From Proposition 2.2, there exists a path $h \in C\left([0,1], H^{1}\right)$ such that $h(0)=U_{1}, h(1)=V_{1}$ and $\Gamma(h(t))<0$ for $t \in[0,1]$. Note that for each $t \in[0,1]$, the function $h(t)$ in the proof of Proposition 2.2 is of the form $t U_{1}\left(s x+x_{0}\right)+t^{\prime} V_{1}\left(s^{\prime} x+x_{0}^{\prime}\right)$ for some $t, t^{\prime}, s, s^{\prime} \in \mathbf{R}$ and $x_{0}, x_{0}^{\prime} \in \mathbf{R}^{n}$. Thus, for each $t \in[0,1]$, the function $h(t)$ is in $C^{\infty} \cap H^{1}$. We let $g(t)$ be the Schwartz symmetrization of $h(t)$. Then, we see that $g(t) \in H_{r}^{1}$ and $\Gamma(g(t))<0$. Since $h(t) \in C^{\infty}\left(\mathbf{R}^{n}\right)$, the function $h(t)$ is co-area regular (see 1 , Definition 1.2.6] for a definition of co-area regularity). Then, by [1, Theorem 1.4], it follows that $g \in C\left([0,1], H_{r}^{1}\right)$. This completes the proof

Let $U$ be a radially symmetric least energy solution of (2). Then, denoting $U_{t}(x)=U\left(\frac{x}{t}\right)$, we see that

$$
\Gamma\left(U_{t}\right)=\left(\frac{t^{n-2}}{2}-\frac{(n-2) t^{n}}{2 n}\right) \int_{\mathbf{R}^{n}}|\nabla U|^{2} d x .
$$


This implies that $I \geq C$. For $u \in H^{1}$, we define

$$
P(u)=\frac{n-2}{2} \int_{\mathbf{R}^{n}}|\nabla u|^{2} d x+n \int_{\mathbf{R}^{n}} \frac{u^{2}}{2}-F(u) d x .
$$

For any $\gamma \in \Psi, P(\gamma(t)) d x>0$ if $t>0$ is small, and

$$
P(\gamma(1))=n \Gamma(\gamma(1))-\int_{\mathbf{R}^{n}}|\nabla \gamma(1)|^{2} d x<0 .
$$

Thus, there exists a $t_{0} \in[0,1]$ such that $P\left(\gamma\left(t_{0}\right)\right)=0$. This implies that $I \leq C$. Thus we conclude the following result.

\section{Proposition 2.4.}

$$
I=C
$$

Let $\mathcal{S}$ be the set of a least energy solution $U$ of (2) satisfying $U(0)=$ $\max _{x \in \mathbf{R}^{n}} U(x)$. Then, we see the following compactness and decay estimate which was proved in [5].

Proposition 2.5. For $N \geq 3, \mathcal{S}$ is compact in $H^{1}\left(\mathbf{R}^{n}\right)$. Moreover, there exist $C, c>0$, independent of $U \in \mathcal{S}$, such that

$$
U(x)+|\nabla U(x)| \leq C \exp (-c|x|)
$$

For $t>0$, the map $x \mapsto \frac{x}{t}$ is a conformal map on $\mathbf{R}^{n}$, which had been used for obtaining the Pohozaev identity (44). For each $t>0$, we define a corresponding map on a ball $B(0, R)$ as follows:

$$
y(x, t)=y_{R}(x, t)=\frac{x}{t+\frac{|x|}{R}(1-t)} .
$$

This map was used in [4]. For each $t \in(0, \infty), y(\cdot, t)$ is a diffeomorphism from $B(0, R)$ onto $B(0, R)$. Moreover, it holds that

$$
\begin{gathered}
\lim _{t \rightarrow 0} y(x, t)=R \frac{x}{|x|} \text { locally uniformly on } \overline{B(0, R)} \backslash\{0\}, \\
y(x, 1)=x
\end{gathered}
$$

and

$$
\lim _{t \rightarrow \infty} y(x, t)=0 \text { uniformly on each compact subset of } B(0, R) .
$$

For any $u \in H_{0}^{1,2}(B(0, R))$, we define

$$
u^{t}(x)=u(y(x, t)), x \in B(0, R), t \in(0, \infty) .
$$

We denote

$$
T=T(t, y, R)=(t-1) \frac{|y|}{R}+1, \quad a_{i j}=a_{i j}(t, y, R)=\frac{T-1}{T} \frac{y_{i} y_{j}}{|y|^{2}} .
$$

The identity $n \times n$-matrix is denoted by $I$. Then, the following estimates were shown in 4].

Proposition 2.6. It follows that for $A=\left(a_{i j}\right)_{i, j=1}^{n}$,

$$
\begin{gathered}
d x=\left(\frac{t}{T}\right)^{n}|\operatorname{det}(I-A)| d y, \\
\left|\frac{d T}{d t}\right| \leq \frac{|y|}{R}, \quad \frac{d^{2} T}{d t^{2}}=0
\end{gathered}
$$


and

$$
\left|\nabla u^{t}(x)\right|^{2}=\frac{1}{t^{2} T^{4}} \sum_{i=1}^{n}\left(T^{2}+\frac{2(T-1) y_{i}^{2}}{|y|^{2}}+\frac{(T-1)^{2} y_{i}^{2}}{T^{2}|y|^{2}}\right)\left(\frac{\partial u}{\partial y_{i}}\right)^{2} .
$$

Moreover, for each $a>1$ and $b \in(1, R]$, there exists some $C>0$ such that

$$
\begin{aligned}
& \max _{|t| \leq a,|y| \leq b}|T(t, y, R)-1| \leq C a b / R, \min _{1 / a \leq t,|y| \leq R} T(t, y, R) \geq 1 / a, \\
& \max _{|y| \leq b}|\operatorname{det}(I-A)-1| \leq C a^{n}|t-1|, \\
& \max _{1 / a \leq t \leq a,|y| \leq b}\left|\frac{d}{d t} \operatorname{det}(I-A)\right| \leq C a^{n+1} \frac{b}{R} \\
& \max _{1 / a \leq t \leq a,|y| \leq b}\left|\frac{d^{2}}{d t^{2}} \operatorname{det}(I-A)\right| \leq C a^{n+2} \frac{b^{2}}{R^{2}}
\end{aligned}
$$

and

$$
\max _{1 / a \leq t \leq a,|y| \leq b}\left|T^{2}+\frac{2(T-1) y_{i}^{2}}{|y|^{2}}+\frac{(T-1)^{2} y_{i}^{2}}{T^{2}|y|^{2}}-1\right| \leq C a^{4} b^{2} / R^{2} .
$$

For $R, D>0$, we consider the following exterior boundary value problem:

$$
\begin{aligned}
\Delta u-u+f(u) & =0, \quad u>0 \quad \text { in } \mathbf{R}^{n} \backslash B(0, R-1), \\
\lim _{|x| \rightarrow \infty} u(x) & =0, \quad u=D \text { on } \partial B(0, R-1) .
\end{aligned}
$$

Proposition 2.7. We assume that $f$ satisfies (f1) and (f2). Fix $R_{0}>0$ and assume that $R>R_{0}$. Then, if $D^{2} R^{N-1}>0$ is sufficiently small, problem (21) has a radially symmetric solution $u \in C^{2}$ satisfying $\frac{d u(r)}{d r} \leq 0$ for $r \geq R-1$.

The proof of Proposition 2.7 above is given in [4, Proposition 3.2].

\section{The Simplest DOMAin CASE}

In this section we will consider the problem (11) when a domain $\Omega$ is a ball. Thus we consider the following problem:

$$
\Delta u-u+f(u)=0, \quad u>0 \text { in } B(0, R), \quad u=0 \text { on } \partial B(0, R) .
$$

We define

$$
H_{r}^{1,2}(B(0, R)) \equiv\left\{u \in H_{0}^{1,2}(B(0, R)) \mid u(x)=u(|x|)\right\}
$$

and

$$
H_{r}^{1,2}\left(\mathbf{R}^{n}\right) \equiv\left\{u \in H_{0}^{1,2}\left(\mathbf{R}^{n}\right) \mid u(x)=u(|x|)\right\} .
$$

We define $\mathcal{S}$ as the set of a least energy solution $U$ of (2) satisfying $U(0)=$ $\max _{x \in \mathbf{R}^{n}} U(x)$. For $u \in H_{r}^{1,2}(B(0, R))$, we define a functional $\Gamma_{R}$ by

$$
\Gamma_{R}(u)=\frac{1}{2} \int_{B(0, R)}|\nabla u|^{2}+u^{2} d x-\int_{B(0, R)} F(u) d x .
$$

For any nonnegative $u \in H^{1,2}\left(\mathbf{R}^{n}\right)$, we let $u^{*}$ be the Schwartz symmetrization of $u$, and define

$$
\mathcal{S}^{*, \delta} \equiv\left\{|U|^{*} \in H_{r}^{1,2}\left(\mathbf{R}^{n}\right) \mid U \in H^{1,2}\left(\mathbf{R}^{n}\right),\|U-\mathcal{S}\| \leq \delta\right\} .
$$


By Proposition 2.5, it follows that for each $\delta \geq 0, \mathcal{S}^{*, \delta}$ is bounded. We find a radially symmetric function $\phi_{R} \in C_{0}^{\infty}\left(\mathbf{R}^{n}\right)$ such that $\phi_{R}(x)=1$ for $|x| \leq R-2$, $\phi_{R}(x)=0$ for $|x| \geq R$ and $\left|\nabla \phi_{R}\right| \leq 1$. We define

$$
\mathcal{S}_{R}^{*, \delta} \equiv\left\{\phi_{R} U^{*} \mid U^{*} \in \mathcal{S}^{\delta}\right\} \subset H_{r}^{1,2}(B(0, R))
$$

and

$$
\mathcal{S}_{R}^{\delta} \equiv\left\{u \in H_{r}^{1,2}(B(0, R)) \mid\left\|U-\mathcal{S}_{R}^{*, \delta}\right\| \leq \delta\right\} .
$$

Here we show the existence of an almost mountain pass solution in $\mathcal{S}_{R}^{\delta}$ for (22) for large $R>0$ when (f1), (f2) and (f3) are satisfied.

Proposition 3.1. We assume that (f1), (f2) and (f3) hold. Suppose that there exists a path $\gamma_{R} \in C\left([0,1], H_{r}^{1,2}(B(0, R))\right)$ and small $\delta>0$ satisfying

(i) $\gamma_{R}(0)=0, \Gamma_{R}\left(\gamma_{R}(1)\right)<0$;

(ii) for some $t_{R} \in(0,1)$,

$$
\begin{aligned}
& \Gamma_{R}\left(\gamma_{R}\left(t_{R}\right)\right)=\max _{t \in[0,1]} \Gamma_{R}\left(\gamma_{R}(t)\right) \equiv C_{R}, \\
& \gamma_{R}\left(t_{R}\right) \in \mathcal{S}_{R}^{\delta} \quad \text { and } \quad \lim _{R \rightarrow \infty} C_{R}=I ;
\end{aligned}
$$

(iii) there exists $\mu>0$ such that for sufficiently large $R>0, \Gamma_{R}\left(\gamma_{R}(t)\right) \leq$ $I-\mu$ if $\gamma_{R}(t) \notin \mathcal{S}_{R}^{\delta}$.

Then, for sufficiently large $R>0$, there exists a radially symmetric positive solution $u_{R} \in C^{2}$ of (22) such that $\Gamma_{R}\left(u_{R}\right) \leq C_{R}$ and $\lim _{R \rightarrow \infty}\left\|u_{R}-\mathcal{S}\right\|=0$. Moreover, for any $\mu>0$, there exist constants $c, C>0$ such that

$$
C_{R} \geq I+c \exp (-2(1+\mu) R)
$$

Remark 3.1. For a radially symmetric solution $U$ of (2), there exists $t_{0}>0$ such that $\Gamma\left(U^{t_{0}}\right)<0$. We define $\gamma_{R}(t)=\phi_{R} U^{t t_{0}}$. Then, the path $\gamma_{R}$ satisfies (i), (ii) and (iii) of Proposition 3.1 (see Proposition 4.1). This implies the existence of a solution $u_{R}$ satisfying the conclusion of Proposition 3.1. An important fact in Proposition 3.1 is that we find a solution $u_{R}$ whose energy $\Gamma_{R}\left(u_{R}\right)$ is less than or equal to the maximum of $\Gamma_{R}$ over any path $\gamma_{R}$ satisfying (i), (ii) and (iii). This fact will be important in proving our main result in the next section.

For any $c \in \mathbf{R}$, we define $\Gamma_{R}^{c} \equiv\left\{u \in H_{r}^{1,2}(B(0, R)) \mid \Gamma_{R}(u) \leq c\right\}$. We will show that for sufficiently large $R>0$, there exists a solution $u_{R} \in \mathcal{S}_{R}^{\delta} \cap \Gamma_{R}^{C_{R}}$ of problem (22). To show this, we need the following lemma.

Lemma 3.1. For sufficiently small $\delta>0$, there exists $\nu>0$ such that for suffciently large $R>0$,

$$
\left|\Gamma_{R}^{\prime}(u)\right| \geq \nu \text { for } u \in \Gamma_{R}^{C_{R}} \cap\left(\mathcal{S}_{R}^{2 \delta}-\mathcal{S}_{R}^{\delta}\right) .
$$

Proof. We regard a member $u$ of $H_{0}^{1,2}(B(0, R))$ as that of $H^{1,2}\left(\mathbf{R}^{n}\right)$ by defining $u(x)=0$ for $|x| \geq R$.

To the contrary, we assume that there exists $u_{R} \in \Gamma_{R}^{C_{R}} \cap\left(\mathcal{S}_{R}^{2 \delta}-\mathcal{S}_{R}^{\delta}\right)$ satisfying $\liminf _{R \rightarrow \infty} \Gamma_{R}^{\prime}\left(u_{R}\right)=0$. Note that $\left\{\mathcal{S}_{R}^{\delta}\right\}_{R}$ is bounded in $H_{r}^{1,2}\left(\mathbf{R}^{n}\right)$. Then, $u_{R}$ converges weakly to some $U \in H_{r}^{1,2}\left(\mathbf{R}^{n}\right)$ along a subsequence as $R \rightarrow \infty$. Without loss of generality, we may assume that $u_{R}$ converges weakly to $U$ in $H_{r}^{1,2}\left(\mathbf{R}^{n}\right)$ as $R \rightarrow \infty$, and $\lim _{R \rightarrow \infty} \Gamma^{\prime}\left(u_{R}\right)=0$. Then, $U$ is a solution of (22). We claim that 
$U \in \mathcal{S} \cap H_{r}^{1,2}\left(\mathbf{R}^{n}\right)$ and $u_{R}$ converges strongly to $U$ in $H_{r}^{1,2}\left(\mathbf{R}^{n}\right)$ as $R \rightarrow \infty$. This contradicts the fact that $u_{R} \notin \mathcal{S}_{R}^{\delta}$, and completes the proof.

Since $\left\{u_{R}\right\}_{R}$ is bounded in $H_{r}^{1,2}\left(\mathbf{R}^{n}\right)$, it follows that for any $D>0$,

$$
\lim _{|z| \rightarrow \infty} \int_{B(z, D)}\left(u_{R}\right)^{2} d x=0 \quad \text { uniformly in } R \text {. }
$$

Then, from the result of Lions (see [20, Lemma I.1]), we see that

$$
\lim _{A \rightarrow \infty} \int_{\mathbf{R}^{n} \backslash B(0, A)}\left(u_{R}\right)^{p+1} d x=0 \quad \text { uniformly in } R .
$$

This implies that $\lim _{R \rightarrow \infty} \int_{\mathbf{R}^{n}} F\left(u_{R}\right) d x=\int_{\mathbf{R}^{n}} F(U) d x$. Then, since $\|U\|^{2} \leq$ $\liminf _{R \rightarrow \infty} \int_{\mathbf{R}^{n}}\left|\nabla u_{R}\right|^{2}+\left(u_{R}\right)^{2} d x$, it follows that $\Gamma(U) \leq C$. By Proposition 2.5, we see that $U \in \mathcal{S} \cap H_{r}^{1,2}\left(\mathbf{R}^{n}\right)$. Since $\Gamma(U)=C$, we deduce that

$$
\|U\|^{2}=\lim _{R \rightarrow \infty} \int_{\mathbf{R}^{n}}\left|\nabla u_{R}\right|^{2}+\left(u_{R}\right)^{2} d x .
$$

This implies that $u_{R}$ converges strongly to $U$ as $R \rightarrow \infty$. This completes the proof.

Proof of Proposition 3.1. Suppose that for large $R>0$, there does not exist a solution $u_{R} \in H_{r}^{1,2}(B(0, R))$ of (22) such that $\Gamma_{R}\left(u_{R}\right) \leq C_{R}$ and $u_{R} \in \mathcal{S}_{R}^{\delta}$. Then, there exists $\nu^{\prime}=\nu^{\prime}(R)>0$ such that $\left|\Gamma^{\prime}(u)\right| \geq \nu^{\prime}$ for $u \in \mathcal{S}_{R}^{\delta}$. Then, by a deformation lemma (see [26]) and Lemma 3.1, we get a path $\gamma_{R}^{\prime}$ such that for large $R>0$, $\max _{t \in[0,1]} \Gamma_{R}\left(\gamma_{R}^{\prime}(t)\right)<I=C, \gamma_{R}^{\prime}(0)=0$ and $\Gamma_{R}\left(\gamma_{R}^{\prime}(1)\right)<0$. This contradicts Proposition 2.1, Proposition 2.3 and Proposition 2.4. Thus, there exists a solution $u_{R} \in H_{r}^{1,2}(B(0, R))$ of (22) such that $\Gamma_{R}\left(u_{R}\right) \leq C_{R}$ and $u_{R} \in \mathcal{S}_{R}^{\delta}$. Then, for some $U \in \mathcal{S}, u_{R}$ converges strongly to $U$ in $H_{r}^{1,2}\left(\mathbf{R}^{n}\right)$ as $R \rightarrow \infty$.

Since we have shown the existence of a solution $u_{R}$ of (22) with $\Gamma_{R}\left(u_{R}\right) \leq$ $C_{R}$, we can follow the proof [4, Proposition 4.3] for the lower energy estimate $C_{R} \geq I+c \exp (-2(1+\mu) R)$. For completeness, we give a detailed proof in the Appendix.

\section{General domain Case}

For any set $A \subset \mathbf{R}^{n}$ and $\varepsilon>0$, we define $A_{\varepsilon} \equiv\left\{x \in \mathbf{R}^{n} \mid \varepsilon x \in A\right\}$. By defining $v(x)=u(\varepsilon x)$, problem (11) is transformed to

$$
\Delta v-v+f(v)=0, \quad u>0 \quad \text { on } \quad \Omega_{\varepsilon}, \quad v=0 \quad \text { on } \quad \partial \Omega_{\varepsilon} .
$$

We denote $H_{\varepsilon} \equiv H_{0}^{1,2}\left(\Omega_{\varepsilon}\right)$. By a translation, we may assume that

$$
\operatorname{dist}(0, \partial \Omega)=\max _{x \in \Omega} \operatorname{dist}(x, \partial \Omega) \text {. }
$$

We define $D \equiv \operatorname{dist}(0, \partial \Omega)$. For $u \in H_{\varepsilon}$, we define

$$
\Gamma_{\varepsilon}(u)=\frac{1}{2} \int_{\Omega_{\varepsilon}}|\nabla u|^{2}+u^{2} d x-\int_{\Omega_{\varepsilon}} F(u) d x,
$$

where $F(t) \equiv \int_{0}^{t} f(s) d s$. Then, a critical point of $\Gamma_{\varepsilon}$ corresponds to a solution of (23). For $u \in H^{1,2}\left(\mathbf{R}^{n}\right)$, we define

$$
\Gamma(u)=\frac{1}{2} \int_{\mathbf{R}^{n}}|\nabla u|^{2}+u^{2} d x-\int_{\mathbf{R}^{n}} F(u) d x .
$$


For small $\varepsilon>0$, we find a function $\chi_{\varepsilon} \in C^{1}\left(\mathbf{R}^{n},[0,1]\right)$ such that $\chi_{\varepsilon}(x)=0$ for $x \notin \Omega_{\varepsilon}, \chi_{\varepsilon}(x)=1$ for $x \in \Omega_{\varepsilon}$ with $\operatorname{dist}\left(x, \partial \Omega_{\varepsilon}\right) \geq 2$ and $\left|\nabla \chi_{\varepsilon}\right| \leq 1$. Let $\mathcal{S}$ be the set of a least energy solution $U$ for (2) satisfying $U(0)=\max _{x \in \mathbf{R}^{n}} U(x)$. We define

$$
\mathcal{S}_{\varepsilon} \equiv\left\{U_{\varepsilon}^{y} \equiv \chi_{\varepsilon} U(\cdot-y) \mid y \in \Omega_{\varepsilon}, \quad U \in \mathcal{S}\right\} .
$$

We fix a radially symmetric monotone decreasing function $U \in \mathcal{S}$, and we find a radially symmetric function $\phi_{\varepsilon} \in C_{0}^{\infty}(B(0, D / \varepsilon),[0,1])$ satisfying $\phi_{\varepsilon}(x)=1$ for $|x| \leq D / \varepsilon-1$ and $\left|\nabla \phi_{\varepsilon}\right| \leq 2$. For $t>0$, we define

$$
U_{\varepsilon}^{t}(x)=\phi_{\varepsilon}(x) U\left(\frac{x}{t}\right), \quad x \in \Omega_{\varepsilon} .
$$

Then, there exists $t_{0}>0$ such that $\Gamma_{\varepsilon}\left(U_{\varepsilon}^{t_{0}}\right)<-1$ for sufficiently small $\varepsilon>0$. Now we see the following upper estimate.

Proposition 4.1. It follows that

$$
\lim _{\varepsilon \rightarrow 0} \Gamma_{\varepsilon}\left(U_{\varepsilon}^{t}\right)=\frac{1}{4}\left(t^{n-2}-\frac{n-2}{n} t^{n}\right) \int_{\mathbf{R}^{n}}|\nabla U|^{2} d x \quad \text { uniformly for } t \in\left(0, t_{0}\right) .
$$

Moreover, for any $\mu \in(0,1)$, there exists $C>0$ such that for small $\varepsilon>0$,

$$
c_{\varepsilon} \equiv \max _{t \in\left[0, t_{0}\right]} \Gamma_{\varepsilon}\left(U_{\varepsilon}^{t}\right) \leq I+C \exp (-2(1-\mu) D / \varepsilon) .
$$

Proof. Here, we will not give a proof. For the proof, see 4, Propositions 4.1, $4.2]$.

For $\delta>0$ and $\mathcal{A} \subset H_{\varepsilon}$, we define

$$
\mathcal{A}^{\delta}=\left\{v \in H_{\varepsilon} \mid \min _{u \in \mathcal{A}}\|v-u\|_{\varepsilon} \leq \delta\right\}
$$

We define

$$
\Gamma_{\varepsilon}^{c} \equiv\left\{u \in H_{\varepsilon} \mid \Gamma_{\varepsilon}(u) \leq c\right\}
$$

We will find a critical point $u_{\varepsilon} \in \mathcal{S}_{\varepsilon}^{\delta} \cap \Gamma_{\varepsilon}^{c_{\varepsilon}}$ for small $\delta, \varepsilon>0$. We prepare some propositions.

Proposition 4.2. For small $\delta>0$, there exist $\alpha>0$ and $\varepsilon_{0}>0$ such that for all $u \in\left(\mathcal{S}_{\varepsilon}^{\delta}-\mathcal{S}_{\varepsilon}^{\delta / 2}\right) \cap \Gamma_{\varepsilon}^{c_{\varepsilon}}$ and $\varepsilon \in\left(0, \varepsilon_{0}\right)$,

$$
\left|\Gamma_{\varepsilon}^{\prime}(u)\right| \geq \alpha
$$

Proof. To the contrary, suppose that there exists a small $\delta>0$ and $u_{\varepsilon} \in\left(\mathcal{S}_{\varepsilon}^{\delta}-\right.$ $\left.\mathcal{S}_{\varepsilon}^{\delta / 2}\right) \cap \Gamma_{\varepsilon}^{c_{\varepsilon}}$ satisfying $\liminf \operatorname{in}_{\varepsilon \rightarrow 0}\left|\Gamma_{\varepsilon}^{\prime}\left(u_{\varepsilon}\right)\right|=0$. For convenience's sake, we may assume that $\lim _{\varepsilon \rightarrow 0}\left|\Gamma_{\varepsilon}^{\prime}\left(u_{\varepsilon}\right)\right|=0$. Then, there exist $\left\{x_{\varepsilon}\right\} \subset \Omega_{\varepsilon}$ and $\left\{U_{\varepsilon}\right\} \subset \mathcal{S}_{\varepsilon}$ such that $\left\|u_{\varepsilon}-\chi_{\varepsilon} U_{\varepsilon}\left(\cdot-x_{\varepsilon}\right)\right\|_{\varepsilon} \leq \delta$. From now on, we regard $u \in H_{\varepsilon}$ as a member in $H^{1}$ by defining $u \equiv 0$ on $\mathbf{R}^{n} \backslash \Omega_{\varepsilon}$.

Suppose that there exist $r>0$ and $y_{\varepsilon} \in \Omega_{\varepsilon}$ satisfying

$$
\lim _{\varepsilon \rightarrow 0}\left|x_{\varepsilon}-y_{\varepsilon}\right|=\infty \quad \text { and } \quad \limsup _{\varepsilon \rightarrow 0} \int_{B\left(y_{\varepsilon}, r\right)}\left(u_{\varepsilon}\right)^{2} d x>0 .
$$

We define $w_{\varepsilon}(x) \equiv u_{\varepsilon}\left(x+y_{\varepsilon}\right)$. Taking a subsequence if it is necessary, we may assume that $w_{\varepsilon}$ converges weakly to a nonnegative function $W$ in $H^{1,2}\left(\mathbf{R}^{n}\right)$ as $\varepsilon \rightarrow 0$ and $\lim _{\varepsilon \rightarrow 0} \int_{B\left(y_{\varepsilon}, r\right)}\left(u_{\varepsilon}\right)^{2} d x>0$. Then, denoting $H_{\xi}^{d} \equiv\left\{x \in \mathbf{R}^{n} \mid\langle x, \xi\rangle>d\right\}$ for $\xi \in S^{n-1}$ and $d \in \mathbf{R} \cup\{-\infty\}$, we see that for some $\xi \in S^{N-1}$ and $d \in \mathbf{R} \cup\{-\infty\}$, $W \in H_{0}^{1,2}\left(H_{\xi}^{d}\right)$ satisfies the following equation:

$$
\Delta W-W+f(W)=0, W>0 \text { in } H_{\xi}^{d} \text { and } W(x)=0 \text { on } \partial H_{\xi}^{d} .
$$


We may assume that $W \in H_{0}^{1,2}\left(\mathbf{R}^{n}\right)$ by defining $W \equiv 0$ on $\mathbf{R}^{n} \backslash H_{\xi}^{d}$. We take $x_{0} \in \partial H_{\xi}^{d}$ if $d \neq-\infty$, and $x_{0}=0$ if $d=-\infty$. Then, since $\nabla W \cdot\left(x-x_{0}\right) \in H_{0}^{1,2}\left(H_{\xi}^{d}\right)$, it follows that

$$
\left.\frac{d \Gamma\left(W\left(t\left(\cdot-x_{0}\right)+x_{0}\right)\right)}{d t}\right|_{t=1}=0 .
$$

Then, through a simple calculation, we see that

$$
\frac{n-2}{2} \int_{\mathbf{R}^{n}}|\nabla W|^{2} d x+n \int_{\mathbf{R}^{n}} \frac{W^{2}}{2}-F(W) d x=0 .
$$

By Proposition 2.1, we see that $\Gamma(W) \geq I$. Note from (25) that $\Gamma(W)=$ $\frac{1}{n} \int_{\mathbf{R}^{n}}|\nabla W|^{2} d x$. This implies that for some large $r>0$,

$$
\lim _{\varepsilon \rightarrow 0} \int_{B\left(y_{\varepsilon}, r\right)}\left|\nabla u_{\varepsilon}\right|^{2}+\left(u_{\varepsilon}\right)^{2} d x>\frac{1}{2 n} \int_{\mathbf{R}^{n}}|\nabla W|^{2} d x \geq \frac{I}{2} .
$$

If $\delta \in\left(0, \sqrt{\frac{I}{2}}\right)$ and $\varepsilon>0$ is sufficiently small, this contradicts the fact that $u_{\varepsilon} \in \mathcal{S}_{R}^{\delta}$. This shows that for any $r>0$ and $y_{\varepsilon} \in \Omega_{\varepsilon}$ with $\lim _{\varepsilon \rightarrow 0}\left|x_{\varepsilon}-y_{\varepsilon}\right|=\infty$,

$$
\limsup _{\varepsilon \rightarrow 0} \int_{B\left(y_{\varepsilon}, r\right)}\left(u_{\varepsilon}\right)^{2} d x=0 .
$$

Then, by [20, Lemma I.1], we see that $\lim _{r \rightarrow \infty} \int_{\mathbf{R}^{n} \backslash B\left(x_{\varepsilon}, r\right)}\left(u_{\varepsilon}\right)^{p+1} d x=0$ uniformly small for $\varepsilon>0$. Now we may assume that $u_{\varepsilon}\left(\cdot+x_{\varepsilon}\right)$ converges weakly to some $U$ in $H^{1,2}\left(\mathbf{R}^{n}\right)$. This implies that

$$
\lim _{\varepsilon \rightarrow 0} \int_{\Omega_{\varepsilon}} F\left(u_{\varepsilon}\right) d x=\int_{\mathbf{R}^{n}} F(U) d x .
$$

Since $\lim _{\varepsilon \rightarrow 0}\left\|u_{\varepsilon}\right\|_{\varepsilon} \geq\|U\|$, it follows that

$$
\liminf _{\varepsilon \rightarrow 0} \Gamma_{\varepsilon}\left(u_{\varepsilon}\right) \geq \Gamma(U) .
$$

Then, for some $\xi \in S^{n-1}$ and $d \in \mathbf{R} \cup\{-\infty\}, U \in H_{0}^{1,2}\left(H_{\xi}^{d}\right)$ satisfies the following equation:

$$
\Delta U-U+f(U)=0, U>0 \text { in } H_{\xi}^{d} \text { and } W(x)=0 \text { on } \partial H_{\xi}^{d} .
$$

As before, we see that

$$
\frac{n-2}{2} \int_{\mathbf{R}^{n}}|\nabla U|^{2} d x+n \int_{\mathbf{R}^{n}} \frac{U^{2}}{2}-F(U) d x=0 .
$$

By Proposition 2.1 we see that $\Gamma(U) \geq C$. Moreover, we see from the maximum principles and Proposition 2.1 that $\Gamma(U)>I$ if $d \neq-\infty$. Then, we see from (26) that $\Gamma(U)=I$. This implies that for some $y_{0} \in \mathbf{R}^{n}, U\left(\cdot+y_{0}\right) \in \mathcal{S}$, $\lim _{\varepsilon \rightarrow 0} \operatorname{dist}\left(x_{\varepsilon}, \partial \Omega_{\varepsilon}\right)=\infty$, and that $\lim _{\varepsilon \rightarrow 0}\left\|u_{\varepsilon}\right\|_{\varepsilon}=\|U\|$. Thus, $u_{\varepsilon}\left(\cdot+x_{\varepsilon}\right)$ converges strongly to $U$ in $H^{1,2}\left(\mathbf{R}^{n}\right)$; this implies that

$$
\lim _{\varepsilon \rightarrow 0}\left\|u_{\varepsilon}-W\left(\cdot-x_{\varepsilon}-y_{0}\right)\right\|_{\varepsilon}=0 \quad \text { for some } \quad W \in \mathcal{S} .
$$

Note that $\lim _{\varepsilon \rightarrow 0} \operatorname{dist}\left(x_{\varepsilon}, \partial \Omega_{\varepsilon}\right)=\infty$; thus $x_{\varepsilon}+y_{0} \in \Omega_{\varepsilon}$ for small $\varepsilon>0$ and $\lim _{\varepsilon \rightarrow 0} \operatorname{dist}\left(x_{\varepsilon}+y_{0}, \partial \Omega_{\varepsilon}\right)=\infty$. This contradicts the fact that $u_{\varepsilon} \notin \mathcal{S}_{\varepsilon}^{\delta / 2}$. This completes the proof.

Proposition 4.3. For sufficiently small $\varepsilon>0$, there exists a critical point of $\Gamma_{\varepsilon}$ in $\mathcal{S}_{\varepsilon}^{\delta} \cap \Gamma_{\varepsilon}^{c_{\varepsilon}}$. 
Proof. Suppose that there exist no critical points of $\Gamma_{\varepsilon}$ in $\mathcal{S}_{\varepsilon}^{\delta} \cap \Gamma_{\varepsilon}^{c_{\varepsilon}}$. Then, by Proposition 4.1 and Proposition 4.2, we deduce via a gradient flow (see [26]) that for small $\sigma>0$, independent of small $\varepsilon>0$, there exists a path $p_{\varepsilon}:[0, t] \rightarrow H_{\varepsilon}$ and $c>0$ satisfying

(i) $p_{\varepsilon}(t)=U_{\varepsilon}^{t}$ for $t \in[0, T] \backslash(1-\sigma, 1+\sigma)$;

(ii) $p_{\varepsilon}(t) \in \mathcal{S}_{\varepsilon}^{\delta}$ for $t \in(1-\sigma, 1+\sigma)$;

(iii) for small $\varepsilon>0, \Gamma_{\varepsilon}\left(p_{\varepsilon}(t)\right) \leq \frac{1}{2} \Gamma(U)-c \delta$.

This contradicts Propositions 2.1, 2.2 and 2.4. This completes the proof.

Proof. Completion of the Main Theorem. Let $u_{\varepsilon}$ be a solution of (23) with

$$
\Gamma_{\varepsilon}\left(u_{\varepsilon}\right) \leq c_{\varepsilon}
$$

and $x_{\varepsilon} \in \Omega_{\varepsilon}$ be a maximum point of $u_{\varepsilon}$. It is standard to see that

$$
0<\liminf _{\varepsilon \rightarrow 0} u_{\varepsilon}\left(x_{\varepsilon}\right)<\limsup _{\varepsilon \rightarrow 0} u_{\varepsilon}\left(x_{\varepsilon}\right)<\infty .
$$

Moreover, as in the proof of Proposition 4.2, taking $\delta \in(0, \sqrt{I / 2})$, we see that $\lim _{\left|y-x_{\varepsilon}\right| \rightarrow \infty} u_{\varepsilon}(x)=0$. Then, it follows from a maximum principle that for any $\delta \in(0,1)$, there exists $C>0$ satisfying $\left|\nabla u_{\varepsilon}(x)\right|+u_{\varepsilon}(x) \leq C \exp \left(-\delta\left|x-x_{\varepsilon}\right|\right)$. Then we deduce from Proposition 2.1 that $u_{\varepsilon}\left(\cdot+x_{\varepsilon}\right)$ converges, up to a subsequence, uniformly to a least energy solution $U \in \mathcal{S}$. Thus, denoting $d_{\varepsilon} \equiv \operatorname{dist}\left(\varepsilon x_{\varepsilon}, \partial \Omega\right)$, we see that $d_{\varepsilon} / \varepsilon=\lim _{\varepsilon \rightarrow 0} \operatorname{dist}\left(x_{\varepsilon}, \partial \Omega_{\varepsilon}\right) \rightarrow \infty$ as $\varepsilon \rightarrow 0$. We will show that $\lim _{\varepsilon \rightarrow 0} d_{\varepsilon}=$ $D$.

From now on, any $u \in H_{0}^{1,2}\left(\Omega^{\prime}\right), \Omega \subset \mathbf{R}^{n}$, is regarded as a member of $H^{1,2}\left(\mathbf{R}^{n}\right)$ by defining $u=0$ on $\mathbf{R}^{n} \backslash \Omega^{\prime}$. By a translation, we may assume that $x_{\varepsilon}=0$. For any $\beta_{1}>\beta_{2}>0$ and $C>2\left(\beta_{1}-\beta_{2}\right)$, we find that $\psi_{\varepsilon} \in C_{0}^{\infty}\left(\mathbf{R}^{n}\right)$ such that

$$
\psi_{\varepsilon}(x)= \begin{cases}1 & \text { if }|x| \leq\left(d_{\varepsilon}+\beta_{2}\right) / \varepsilon \\ 0 & \text { if }|x| \geq\left(d_{\varepsilon}+\beta_{1}\right) / \varepsilon\end{cases}
$$

$0 \leq \psi_{\varepsilon}(x) \leq 1,\left|\nabla \psi_{\varepsilon}\right| \leq C \varepsilon$ and $\left|\nabla^{2} \psi_{\varepsilon}\right| \leq C \varepsilon^{2}$. Let $\tilde{\Omega}_{\varepsilon}=B\left(0, \frac{d_{\varepsilon}+\beta_{1}}{\varepsilon}\right) \cap \Omega_{\varepsilon}$. Then, there exists $\beta_{3}=\beta_{3}(\varepsilon) \in\left(0, \beta_{1}\right)$ satisfying $\left|\tilde{\Omega}_{\varepsilon}\right|=\left|B\left(0, \frac{d_{\varepsilon}+\beta_{3}}{\varepsilon}\right)\right|$. Since $\partial \Omega \in C^{2}$, it follows that $\left\{\beta_{3}(\varepsilon)\right\}_{\varepsilon}$ is bounded away from 0 and $\beta_{1}$. We take $\beta_{2}>0$ so that $\beta_{3}<\beta_{2}<\beta_{1}$. From the decay estimate of $u_{\varepsilon}$, there exists some $C^{\prime}>0$ such that

$$
\left|\Gamma_{\varepsilon}\left(u_{\varepsilon}\right)-\Gamma_{\varepsilon}\left(u_{\varepsilon} \psi_{\varepsilon}\right)\right| \leq C^{\prime} \exp \left(-\frac{2 \delta}{\varepsilon}\left(d_{\varepsilon}+\beta_{2}\right)\right)
$$

We define $z_{\varepsilon}=u_{\varepsilon} \psi_{\varepsilon}$ and $z_{\varepsilon}^{t}(x)=z_{\varepsilon}(y(x, t))$ with

$$
y(x, t)=x /\left(t+\frac{\varepsilon|x|}{d_{\varepsilon}+\beta_{1}}(1-t)\right) .
$$


Since $\Delta z_{\varepsilon}-z_{\varepsilon}+f\left(z_{\varepsilon}\right)=0$ in $\Omega_{\varepsilon} \cap B\left(0, \frac{d_{\varepsilon}+\beta_{2}}{\varepsilon}\right)$, it follows that

$$
\begin{aligned}
& \left.\frac{d \Gamma\left(z_{\varepsilon}^{t}\right)}{d t}\right|_{t=1} \nabla z_{\varepsilon} \cdot \nabla\left(x \cdot \nabla z_{\varepsilon} \frac{\varepsilon|x|-d_{\varepsilon}-\beta_{1}}{d_{\varepsilon}+\beta_{1}}\right) d x \\
= & \int_{B\left(0, \frac{d_{\varepsilon}+\beta_{1}}{\varepsilon}\right) \cap \Omega_{\varepsilon}}\left(z_{\varepsilon}-f\left(z_{\varepsilon}\right)\right)\left(x \cdot \nabla z_{\varepsilon} \frac{\varepsilon|x|-d_{\varepsilon}-\beta_{1}}{d_{\varepsilon}+\beta_{1}}\right) d x \\
& +\int_{B\left(0, \frac{d_{\varepsilon}+\beta_{1}}{\varepsilon}\right) \cap \Omega_{\varepsilon}} \frac{\partial z_{\varepsilon}}{\partial \nu} x \cdot \nabla z_{\varepsilon} \frac{\varepsilon|x|-d_{\varepsilon}-\beta_{1}}{d_{\varepsilon}+\beta_{1}} d \sigma \\
= & \int_{\partial\left(B\left(0, \frac{d_{\varepsilon}+\beta_{2}}{\varepsilon}\right) \cap \Omega_{\varepsilon}\right)} \nabla z_{\varepsilon} \cdot \nabla\left(x \cdot \nabla z_{\varepsilon} \frac{\varepsilon|x|-d_{\varepsilon}-\beta_{1}}{d_{\varepsilon}+\beta_{1}}\right) d x \\
& +\int_{B\left(0, \frac{d_{\varepsilon}+\beta_{1}}{\varepsilon}\right) \cap \Omega_{\varepsilon} \backslash B\left(0, \frac{d_{\varepsilon}+\beta_{2}}{\varepsilon}\right)}\left(z_{\varepsilon}-f\left(z_{\varepsilon}\right)\right)\left(x \cdot \nabla z_{\varepsilon} \frac{\varepsilon|x|-d_{\varepsilon}-\beta_{1}}{d_{\varepsilon}+\beta_{1}}\right) d x, \\
& +\int_{B\left(0, \frac{d_{\varepsilon}+\beta_{1}}{\varepsilon}\right) \cap \Omega_{\varepsilon} \backslash B\left(0, \frac{d_{\varepsilon}+\beta_{2}}{\varepsilon}\right)}
\end{aligned}
$$

where $\nu$ and $d \sigma$ are the outward normal and the volume element on $\partial\left(B\left(0, \frac{d_{\varepsilon}+\beta_{2}}{\varepsilon}\right) \cap \Omega_{\varepsilon}\right)$, respectively. From the $W^{2, p}$ estimates, [16, Theorem 9.13] and the decay estimates of $u_{\varepsilon}$, we deduce that for any $\delta \in(0,1)$, there exists some $C>0$ satisfying

$$
\left|\frac{d \Gamma\left(z_{\varepsilon}^{t}\right)}{d t}\right|_{t=1} \leq C \exp \left(-\frac{2 \delta}{\varepsilon}\left(d_{\varepsilon}+\beta_{3}\right)\right)
$$

Denoting

$$
T_{\varepsilon}=T_{\varepsilon}(y) \equiv(t-1) \frac{\varepsilon|y|}{d_{\varepsilon}+\beta_{1}}+1 \quad \text { and } \quad A=A_{\varepsilon} \equiv\left(\frac{T-1}{T} \frac{y_{i} y_{j}}{|y|^{2}}\right),
$$

we deduce from Proposition 2.6 that

$$
\begin{gathered}
\int_{\mathbf{R}^{n}}\left|\nabla z_{\varepsilon}^{t}\right|^{2} d x \\
=t^{n-2} \sum_{i=1}^{n} \int_{\mathbf{R}^{n}}\left|\frac{\partial z_{\varepsilon}}{\partial y_{i}}\right|^{2}\left|T^{2}+\frac{2(T-1) y_{i}^{2}}{|y|^{2}}+\frac{(T-1)^{2} y_{i}^{2}}{T^{2}|y|^{2}}\right| \frac{|\operatorname{det}(I-A)|}{T^{n+4}} d y, \\
\int_{\mathbf{R}^{n}}\left|z_{\varepsilon}^{t}\right|^{2} d x=t^{n} \int_{\mathbf{R}^{n}}\left|z_{\varepsilon}\right|^{2} \frac{|\operatorname{det}(I-A)|}{T^{n}} d y
\end{gathered}
$$

and

$$
\int_{\mathbf{R}^{n}} F\left(z_{\varepsilon}^{t}\right) d x=t^{n} \int_{\mathbf{R}^{n}} F\left(z_{\varepsilon}\right) \frac{|\operatorname{det}(I-A)|}{T^{n}} d y .
$$

Note that for fixed $a, b>1, \lim _{\varepsilon \rightarrow 0} T_{\varepsilon}=1$ and $\lim _{\varepsilon \rightarrow 0} A_{\varepsilon}=0$ uniformly for $t \in(1 / a, a)$ and $|y| \leq b$. From the exponential decays of $z_{\varepsilon}$ and $U$, we deduce that if $a>1$ is fixed,

$$
\begin{aligned}
& \lim _{\varepsilon \rightarrow 0} \int_{\mathbf{R}^{n}}\left|\nabla z_{\varepsilon}^{t}\right|^{2} d x=t^{n-2} \int_{\mathbf{R}^{n}}|\nabla U|^{2} d x, \\
& \lim _{\varepsilon \rightarrow 0} \int_{\mathbf{R}^{n}}\left|z_{\varepsilon}^{t}\right|^{2} d x=t^{n} \int_{\mathbf{R}^{n}} U^{2} d x, \\
& \lim _{\varepsilon \rightarrow 0} \int_{\mathbf{R}^{n}} F\left(z_{\varepsilon}^{t}\right) d x=t^{n} \int_{\mathbf{R}^{n}} F(U) d x
\end{aligned}
$$


uniformly for $t \in(1 / a, a)$. Then, it follows from the Pohozaev identity (4) that

$$
\lim _{\varepsilon \rightarrow 0} \Gamma\left(z_{\varepsilon}^{t}\right)=\left(\frac{t^{n-2}}{2}-\frac{n-2}{2 n} t^{n}\right) \int_{\mathbf{R}^{n}}|\nabla U|^{2} d x
$$

uniformly for $t \in(1 / a, a)$. Moreover, differentiating both sides of (31), (32), (33) and using Proposition 2.6. we see that for sufficiently small $\gamma>0$,

$$
\lim _{\varepsilon \rightarrow 0} \frac{d^{2} \Gamma\left(z_{\varepsilon}^{t}\right)}{d t^{2}} \leq-\frac{n-2}{2} \int_{\mathbf{R}^{n}}|\nabla U|^{2} d x \quad \text { for } \quad t \in(1-\gamma, 1+\gamma) .
$$

This and (30) implies that there exists $t_{\varepsilon}>0$ satisfying

$$
\Gamma\left(z_{\varepsilon}^{t_{\varepsilon}}\right)=\max _{t \in(1-\gamma, 1+\gamma)} \Gamma\left(z_{\varepsilon}^{t}\right) \text { and }\left|t_{\varepsilon}-1\right| \leq C \exp \left(-\frac{2 \delta}{\varepsilon}\left(d_{\varepsilon}+\beta_{3}\right)\right)
$$

for some $C>0$. Then, through the Taylor expansion as in (46), we deduce that for small $\gamma>0$,

$$
\left|\Gamma_{\varepsilon}\left(z_{\varepsilon}\right)-\max _{t \in[1-\gamma, 1+\gamma]} \Gamma_{\varepsilon}\left(z_{\varepsilon}^{t}\right)\right| \leq C \exp \left(-\frac{4 \delta}{\varepsilon}\left(d_{\varepsilon}+\beta_{3}\right)\right) .
$$

By (f1), (34) and (35), we see that for some small $t_{0}>0$,

$$
\frac{d \Gamma\left(s z_{\varepsilon}^{t_{0}}\right)}{d s} \geq 0 \quad \text { for } s \in[0,1] \text { and small } \varepsilon>0 .
$$

Moreover, by (34), (35) and (36), we see that for some $t_{1}>0$,

$$
\Gamma\left(z_{\varepsilon}^{t_{1}}\right)<-1 \quad \text { for small } \varepsilon>0 .
$$

Then, we define a continuous curve $\alpha_{\varepsilon}(t):[0,1] \rightarrow H_{0}^{1,2}\left(\mathbf{R}^{n}\right)$ by

$$
\alpha_{\varepsilon}(t)= \begin{cases}2 t z_{\varepsilon}^{t_{0}}, & t \in[0,1 / 2], \\ z_{\varepsilon}^{(2-2 t) t_{0}+(2 t-1) t_{1}}, & t \in[1 / 2,1] .\end{cases}
$$

From (37), we see that for sufficiently small $\varepsilon>0$,

$$
\max _{t \in[0,1]} \Gamma_{\varepsilon}\left(\alpha_{\varepsilon}(t)\right)=\max _{t \in[1 / 2,1]} \Gamma_{\varepsilon}\left(\alpha_{\varepsilon}(t)\right)=\max _{t \in\left[t_{0}, t_{1}\right]} \Gamma_{\varepsilon}\left(z_{\varepsilon}^{t}\right)=\max _{t \in[1-\gamma, 1+\gamma]} \Gamma_{\varepsilon}\left(z_{\varepsilon}^{t}\right) .
$$

Thus, it follows that

$$
\max _{t \in[0,1]} \Gamma_{\varepsilon}\left(\alpha_{\varepsilon}(t)\right) \leq \Gamma_{\varepsilon}\left(z_{\varepsilon}\right)+C \exp \left(-\frac{4 \delta}{\varepsilon}\left(d_{\varepsilon}+\beta_{3}\right)\right) .
$$

Since $\lim _{\varepsilon \rightarrow 0} c_{\varepsilon}=I=C$, it follows that for any small $\alpha>0$, there exists a constant $\beta>0$ such that

$$
\limsup _{\varepsilon \rightarrow 0} \max _{t \in\left[0, t_{1}\right] \backslash(1-\beta, 1+\beta)} \Gamma\left(z_{\varepsilon}^{t}\right)<I-\alpha .
$$

We let $S_{\varepsilon}^{t}$ be the Schwartz symmetrization of $\alpha_{\varepsilon}(t)$ for each $t>0$. Then,

$$
S_{\varepsilon}^{t} \in H_{0}^{1,2}\left(B\left(0, \frac{d_{\varepsilon}+\beta_{3}}{\varepsilon}\right)\right) \text { and } S_{\varepsilon}^{0}=0, \quad \Gamma\left(S_{\varepsilon}^{t_{1}}\right)<0 .
$$

By a rearrangement argument, we see that

$$
\max _{t \in\left[0, t_{1}\right]} \Gamma\left(\alpha_{\varepsilon}(t)\right) \geq \max _{t \in\left[0, t_{1}\right]} \Gamma\left(S_{\varepsilon}^{t}\right) \text { and } \limsup _{\varepsilon \rightarrow 0} \max _{t \in\left[0, t_{1}\right] \backslash(1-\beta, 1+\beta)} \Gamma\left(S_{\varepsilon}^{t}\right)<I-\alpha .
$$

Moreover, for any small $\delta>0$, there exists $\varepsilon_{0}>0$ such that for $\varepsilon \in\left(0, \varepsilon_{0}\right)$,

$$
S_{\varepsilon}^{1}=\left(z_{\varepsilon}\right)^{*} \in \mathcal{S}_{\left(d_{\varepsilon}+\beta_{3}\right) / \varepsilon}^{\delta / 2} .
$$


It is easy to see that $S_{\varepsilon}^{t}$ is continuous with respect to $t \in\left[0, t_{1}\right]$. Thus, $S_{\varepsilon}^{t} \in \mathcal{S}_{\left(d_{\varepsilon}+\beta_{3}\right) / \varepsilon}^{\delta}$ if $|t-1|$ is sufficiently small. Then, by Proposition [3.1, there exists a solution $w_{\varepsilon}$ of (22) with $R=\left(d_{\varepsilon}+\beta_{3}\right) / \varepsilon$ such that

$$
\Gamma\left(w_{\varepsilon}\right) \leq \max _{t \in\left[0, t_{1}\right]} \Gamma\left(S_{\varepsilon}^{t}\right),
$$

and that for some $C>0$,

$$
\Gamma\left(w_{\varepsilon}\right) \geq I+\exp \left(-\frac{2 \delta_{1}}{\varepsilon}\left(d_{\varepsilon}+\beta_{3}\right)\right) .
$$

Thus we conclude that for any $\delta_{1}>1>\delta_{2}$, there exist $C_{1}, C_{2}, C_{3}>0$ satisfying

$$
\begin{aligned}
\Gamma_{\varepsilon}\left(u_{\varepsilon}\right) \geq & \Gamma_{\varepsilon}\left(z_{\varepsilon}\right)-C_{1} \exp \left(-\frac{2 \delta}{\varepsilon}\left(d_{\varepsilon}+\beta_{2}\right)\right) \\
\geq & \Gamma(U)+C_{2} \exp \left(-\frac{2 \delta_{1}}{\varepsilon}\left(d_{\varepsilon}+\beta_{3}\right)\right) \\
& \quad C_{1} \exp \left(-\frac{2 \delta}{\varepsilon}\left(d_{\varepsilon}+\beta_{2}\right)\right)-C_{3} \exp \left(-\frac{4 \delta_{2}}{\varepsilon}\left(d_{\varepsilon}+\beta_{3}\right)\right) .
\end{aligned}
$$

Thus, it follows that for any $\beta>0$, there exists $C>0$ satisfying

$$
c_{\varepsilon} \geq \Gamma_{\varepsilon}\left(u_{\varepsilon}\right) \geq I+C \exp \left(-\frac{2}{\varepsilon}\left(d_{\varepsilon}+\beta\right)\right) \text {. }
$$

Then, by Proposition 4.1 we conclude that $\lim _{\varepsilon \rightarrow 0} d_{\varepsilon}=D$. It is standard to see other properties of $u_{\varepsilon}$. This completes the proof.

\section{Appendix: A lower energy estimate in Proposition 3.1}

Now we show the lower estimate: for any $\mu \in(0,1)$, there exists $c>0$ such that $C_{R} \geq \Gamma(U)+c \exp (-2(1+\mu) R)$.

Since $\left\{\mathcal{S}_{R}^{\delta}\right\}_{R>1}$ is bounded, we see that

$$
\left\{\int_{B(0, R)}\left|\nabla u_{R}\right|^{2}+\left(u_{R}\right)^{2} d x\right\}_{R>1}
$$

is bounded. Then, since $u_{R}$ is radially symmetric, it follows that $\lim _{x \rightarrow \infty} u_{R}(x)$ $=0$ uniformly for $R>1$. Then, by the comparison principle, we see that for any $\gamma \in(0,1)$, there exists a constant $C>0$, independent of $R>1$, satisfying

$$
u_{R}(x) \leq C \exp (-\gamma|x|), \quad x \in B(0, R) .
$$

Then, we see from the elliptic estimates (refer to [16]) that for any $\gamma \in(0,1)$, there exists $C^{\prime}>0$ satisfying

$$
\left|\frac{d u_{R}}{d r}\right| \leq C^{\prime} \exp (-\gamma E) .
$$

We may assume that $u_{R}$ converges weakly to some $U$ in $H^{1,2}\left(\mathbf{R}^{n}\right)$ as $R \rightarrow \infty$. It is easy to see that $U$ is a solution of (2). Then, we deduce from Proposition 2.4 and the above estimates that $U$ is a least energy solution of (2) and $u_{R}$ converges uniformly to $U$ as $R \rightarrow \infty$. For $\gamma \in(0,1)$ and $C>0$, we define

$$
\psi_{R}(x)=C(\exp (-|x|(1+\gamma))-\exp (-R(1+\gamma))) .
$$


Then we see that

$$
\begin{aligned}
& \Delta \psi_{R}-\psi_{R}+\frac{f\left(u_{R}\right)}{u_{R}} \psi_{R} \\
& =\left((1+\gamma)^{2}-\frac{(n-1)(1+\gamma)}{r}-1+\frac{f\left(u_{R}\right)}{u_{R}}\right) C \exp (-(1+\gamma)|x|) \\
& \quad+\left(1-\frac{f\left(u_{R}\right)}{u_{R}}\right) C \exp (-R(1+\gamma)) .
\end{aligned}
$$

By (38), there exists $R_{0}>0$, independent of large $R$, such that

$$
\Delta \psi_{R}-\psi_{R}+\frac{f\left(u_{R}\right)}{u_{R}} \psi_{R} \geq 0 \text { for } R_{0} \leq|x|<R .
$$

We take a constant $c^{\prime}>0$ such that $u_{R}\left(R_{0}\right) \geq c^{\prime} \psi_{R}\left(R_{0}\right)$ for large $R$. Then, by the comparison principle, we see that $u_{R}(r) \geq c^{\prime} \psi_{R}(r)$ for $r \in\left(r_{0}, R\right)$. In particular, we see that

$$
u_{R}(R-1) \geq c^{\prime} \psi_{R}(R-1)=c^{\prime} \exp (-R(1+\gamma))(\exp (1+\gamma)-1) .
$$

Thus, for any $\gamma \in(0,1)$, there exist $c, C>0$ such that

$$
c \exp (-R(1+\gamma)) \leq u_{R}(R-1) \leq C \exp (-R(1-\gamma)) .
$$

Then, from Proposition 2.7 we see that for sufficiently large $R>1$, there exists a radially symmetric decreasing solution $V_{R}$ of

$$
\begin{aligned}
\Delta u-u+f(u) & =0 \quad \text { on } \quad \mathbf{R}^{n} \backslash \overline{B(0, R-1)}, \\
u(R-1) & =u_{R}(R-1) \quad \text { and } \lim _{x \rightarrow \infty} u(x)=0 .
\end{aligned}
$$

Then, from the comparison principle and the decay property (40), we deduce that for any $\gamma \in(0,1)$, there exists $C>0$ satisfying

$$
\left|V_{R}(r)\right|+\left|\frac{d V_{R}(r)}{d r}\right| \leq C \exp (-\gamma r) \text { for } \quad r \geq R-1
$$

Define

$$
w_{R}(x) \equiv \begin{cases}u_{R}(x) & \text { for }|x| \leq R-1 \\ V_{R}(x) & \text { for }|x| \geq R-1\end{cases}
$$

and $w_{R}^{t}(x) \equiv w_{R}\left(\frac{x}{t}\right)$. Since the least energy solution $U$ is a mountain pass solution of (2) (see [18]) and $w_{R}$ converges uniformly to $U$, it follows that

$$
\max _{t \in(0, \infty)} \Gamma\left(w_{R}^{t}\right) \geq \Gamma(U)
$$

We define

$$
u_{R}^{t}(x)= \begin{cases}u_{R}\left(\frac{x}{t}\right) & \text { for } t>0, x \in B(0, t R), \\ 0 & \text { for } t>0, x \in \mathbf{R}^{n} \backslash B(0, t R) .\end{cases}
$$

From (4) and a change of variables, we see that as $R \rightarrow \infty$,

$$
\begin{aligned}
& \Gamma\left(u_{R}^{t}\right) \\
& =\frac{t^{n-2}}{2} \int_{B(0, R)}\left|\nabla u_{R}\right|^{2} d x+\frac{t^{n}}{2} \int_{B(0, R)}\left|u_{R}\right|^{2}-2 F\left(u_{R}\right) d x \\
& \rightarrow \frac{t^{n-2}}{2} \int_{\mathbf{R}^{n}}|\nabla U|^{2} d x+\frac{t^{n}}{2} \int_{\mathbf{R}^{n}} U^{2}-2 F(U) d x \\
& =\left(\frac{t^{n-2}}{2}-\frac{(n-2) t^{n}}{2 n}\right) \int_{\mathbf{R}^{n}}|\nabla U|^{2} d x
\end{aligned}
$$


uniformly for $t \in(0, \infty)$. We take $s_{R}>0$ satisfying

$$
\Gamma\left(u_{R}^{s_{R}}\right)=\max _{t \in(0, \infty)} \Gamma\left(u_{R}^{t}\right) .
$$

Then, it is easy to see that $\lim _{R \rightarrow \infty} s_{R}=1$. Then, by the Pohozaev identity (4), we see that as $R \rightarrow \infty$,

$$
\begin{aligned}
& \frac{d^{2} \Gamma\left(u_{R}^{t}\right)}{d t^{2}} \\
= & (n-2)(n-3) \frac{t^{n-4}}{2} \int_{B(0, R)}\left|\nabla u_{R}\right|^{2} d x \\
& +n(n-1) \frac{t^{n-2}}{2} \int_{B(0, R)}\left|u_{R}\right|^{2} d x-n(n-1) t^{n-2} \int_{B(0, R)} F\left(u_{R}\right) d x \\
\rightarrow & (n-2)(n-3) \frac{t^{n-4}}{2} \int_{\mathbf{R}^{n}}|\nabla U|^{2} d x+n(n-1) \frac{t^{n-2}}{2} \int_{\mathbf{R}^{n}} U^{2}-2 F(U) d x \\
= & \frac{n-2}{2} \int_{\mathbf{R}^{n}}|\nabla U|^{2} d x\left((n-3) t^{n-4}-(n-1) t^{n-2}\right) .
\end{aligned}
$$

Thus, there exist $C>0, t^{\prime} \in(0,1)$ and $R_{0}>0$ such that

$$
\frac{d^{2} \Gamma\left(u_{R}^{t}\right)}{d t^{2}}<-C \text { for } R \geq R_{0} \text { and } t \in\left(1-t^{\prime}, 1+t^{\prime}\right) .
$$

Let $\omega_{n-1}$ be the volume of $S^{n-1}$. Then we see that

$$
\begin{aligned}
& \left.\frac{d \Gamma\left(u_{R}^{t}\right)}{d t}\right|_{t=1} \\
& =-\int_{B(0, R)} \nabla u_{R} \nabla\left(x \cdot \nabla u_{R}\right)+u_{R}\left(x \cdot \nabla u_{R}\right)-f\left(u_{R}\right)\left(x \cdot \nabla u_{R}\right) d x \\
& =-\omega_{n-1}(R)^{N}\left(\frac{d u_{R}(R)}{d r}\right)^{2} .
\end{aligned}
$$

Then, by (39), we see that for any $\gamma \in(0,1)$, there exists $C>0$ satisfying

$$
\left|\frac{d \Gamma\left(u_{R}^{t}\right)}{d t}\right|_{t=1} \leq C \exp (-2 \gamma R) .
$$

From (42), (43) and (44), we deduce that there exists $D>0$ satisfying

$$
\left|1-s_{R}\right| \leq D \exp (-2 \gamma R) \text {. }
$$

Note that

$$
\Gamma\left(u_{R}^{s_{\varepsilon}}\right)=\Gamma\left(u_{R}^{1}\right)+\left.\frac{d \Gamma\left(u_{R}^{t}\right)}{d t}\right|_{t=1}\left(s_{R}-1\right)+O\left(\left|s_{R}-1\right|^{2}\right) .
$$

Thus, we see that for some $C^{\prime}>0$,

$$
\Gamma\left(u_{R}^{t}\right) \leq \Gamma\left(u_{R}\right)+C^{\prime} \exp (-3 R), \quad t \in(0, \infty) .
$$

We define $V_{R}^{t}(x) \equiv V_{R}\left(\frac{x}{t}\right)$ for $t>0$, and $e(v) \equiv \frac{1}{2}|\nabla v|^{2}+\frac{1}{2} v^{2}-F(v)$. Then we see that

$$
\begin{aligned}
& \Gamma\left(u_{R}^{t}\right) \\
& =\Gamma\left(w_{R}^{t}\right)+\int_{B(0, t R) \backslash B(0, t(R-1))} e\left(u_{R}^{t}\right) d x-\int_{\mathbf{R}^{n} \backslash B(0, t(R-1))} e\left(V_{R}^{t}\right) d x \\
& \equiv A(t)+B(t)-C(t) .
\end{aligned}
$$


We take $t(R)>0$ so that $A(t(R))=\max _{t \in(0, \infty)} A(t)$. Since $U$ is a mountain pass solution (see $([18]))$, it follows that

$$
A(t(R)) \geq \Gamma(U) .
$$

Since $w_{R}$ converges to a least energy solution $U$ of (2), it follows that $\lim _{R \rightarrow \infty} t(R)=$ 1. By (41) and a similar manner as in (45), we deduce that for any $\gamma \in(0,1)$, there exists $C>0$ satisfying $|t(R)-1| \leq C \exp (-2 \gamma R)$. Moreover, from (40) and (41), we deduce that for any $\gamma \in(0,1)$, there exists $C>0$ satisfying

$$
\left|B^{\prime}(1)-C^{\prime}(1)\right| \leq C \exp (-2 \gamma R) .
$$

This implies that for some $C>0$,

$$
|(B(t(R))-C(t(R)))-(B(1)-C(1))| \leq C \exp (-3 R) .
$$

Note that

$$
\int_{B(0, R) \backslash B(0, R-1)} u_{R} \Delta u_{R}-\left(u_{R}\right)^{2}+f\left(u_{R}\right) u_{R} d x=0
$$

and

$$
\int_{\mathbf{R}^{n} \backslash B(0, R-1)} V_{R} \Delta V_{R}-\left(V_{R}\right)^{2}+f\left(V_{R}\right) V_{R} d x=0 .
$$

Since $\lim _{t \rightarrow 0} F(t) / t^{2}=\lim _{t \rightarrow 0} f(t) / t=0$, it follows from integration by parts that

$$
\begin{aligned}
B(1)= & \frac{1}{2} \int_{B(0, R) \backslash B(0, R-1)}\left|\nabla u_{R}\right|^{2}+\left(u_{R}\right)^{2}-f\left(u_{R}\right) u_{R} d x \\
& +\int_{B(0, R) \backslash B(0, R-1)} \frac{1}{2} f\left(u_{R}\right) u_{R}-F\left(u_{R}\right) d x \\
= & -\frac{1}{2} \omega_{n-1}(R-1)^{n-1} u_{R}(R-1) \frac{d u_{R}(R-1)}{d r} \\
& +\omega_{n-1}(R-1)^{n-1} o\left(\left(u_{R}(R-1)\right)^{2}\right) .
\end{aligned}
$$

By a similar manner, we deduce that

$$
C(1)=-\frac{1}{2}(R-1)^{n-1} V_{R}(R-1) \frac{d V_{R}(R-1)}{d r}+\omega_{N-1}(R-1)^{n-1} o\left(\left(V_{R}(R-1)\right)^{2}\right) .
$$

Thus, we see that

$$
\begin{aligned}
& B(1)-C(1) \\
& \geq \omega_{N-1}(R-1)^{n-1} u_{R}(R-1)\left(\frac{1}{2} \frac{d V_{R}(R-1)}{d r}-\frac{1}{2} \frac{d u_{R}(R-1)}{d r}\right) \\
& +\omega_{N-1}(R-1)^{n-1} o\left(\left(u_{R}(R-1)\right)^{2}\right) .
\end{aligned}
$$

We define

and

$$
\tilde{V}_{R}(r) \equiv V_{R}(r+R-1) / u_{R}(R-1)
$$

Then we see that

$$
\frac{d^{2} \tilde{V}_{R}}{d r^{2}}+\frac{n-1}{r+R-1} \frac{d \tilde{V}_{R}}{d r}-\tilde{V}_{R}+\frac{1}{u_{R}(R-1)} f\left(u_{R}(R-1) \tilde{V}_{R}\right)=0, r>0,
$$

and

$$
\frac{d^{2} \tilde{u}_{R}}{d r^{2}}+\frac{n-1}{r+R-1} \frac{d \tilde{u}_{R}}{d r}-\tilde{u}_{R}+\frac{1}{u_{R}(R-1)} f\left(u_{R}(R-1) \tilde{u}_{R}\right)=0, r \in(0,1) .
$$


Since $\lim _{s \rightarrow 0} \frac{f(s)}{s}=0$, it is standard to show from Proposition 2.7 that $\tilde{V}_{R}$ converges locally uniformly on $[0, \infty)$ in $C^{1}$-sense to a unique positive function $V_{1}$ satisfying

$$
\frac{d^{2} V_{1}}{d r^{2}}(r)-V_{1}(r)=0, r \in(0, \infty), \quad V_{1}(0)=1, V_{1}(r) \leq 1 .
$$

Likewise, $\tilde{u}_{R}$ converges uniformly on $[0,1]$ in $C^{1}$-sense to a unique positive function $V_{2}$ satisfying

$$
\frac{d^{2} V_{2}}{d r^{2}}(r)-V_{2}(r)=0, r \in(0,1), \quad V_{2}(0)=1, V_{2}(1)=0 .
$$

It is obvious that

$$
\frac{d V_{1}(0)}{d t}-\frac{d V_{2}(0)}{d t}>0
$$

This implies that for some $C>0$,

$$
\frac{d U_{R}(R-1)}{d r}-\frac{d u_{R}(R-1)}{d r} \geq C u_{R}(R-1)
$$

if $R>0$ is sufficiently large. Then, we see from (50) that there exists $C>0$ satisfying

$$
B(1)-C(1) \geq C(R-1)^{n-1}\left(u_{R}(R-1)\right)^{2} \quad \text { for large } \quad R>0 .
$$

Then we see from (40) and (49) that for any $\delta>1$, there exists a constant $C>0$ satisfying

$$
B(t(R))-C(t(R)) \geq C \exp (-2 \delta R)
$$

Combining this with (47) and (48), we deduce that for any $\mu>0$, there exists a constant $C>0$ satisfying

$$
\Gamma\left(u_{R}\right) \geq \Gamma(U)+C \exp (-2(1+\mu) R) .
$$

This completes the proof.

\section{REFERENCES}

[1] F. J. Almgren and E.H. Lieb, Symmetric decreasing rearrangement is sometimes continuous, J. Amer. Math. Soc., 2 (1989), 683-773. MR1002633 (90f:49038)

[2] A. Ambrosetti And P.H. Rabinowitz, Dual variational methods in critical point theory and applications, J. Functional Analysis, 14 (1973), 349-381. MR0370183 (51:6412)

[3] J. Byeon, Existence of large positive solutions of some nonlinear elliptic equations on singularly perturbed domains, Comm. in Partial Differential Equations, 22 (1997), 1731-1769. MR.1469588 (98i:35057)

[4] J. BYEON, Mountain pass solutions for singularly perturbed nonlinear Dirichlet problems, J. Differential Equations, 217 (2005), 257-281. MR2168823 (2006k:35081)

[5] J Byeon and L. Jeanjean, Standing waves for nonlinear Schrodinger equations with a general nonlinearity, Arch. Rational Mech. Anal., 185 (2007), 185-200. MR2317788

[6] J Byeon and L. Jeanjean, Multi-peak standing waves for nonlinear Schrodinger equations with a general nonlinearity, Discrete Contin. Dyn. Syst., 19 (2007), 255-269. MR2335747

[7] J Byeon, L. Jeanjean and K. Tanaka, Standing waves for nonlinear Schrodinger equations with a general nonlinearity: one and two dimensional cases, Comm. Partial Differential Equations, 33 (2008), 1113-1136. MR.2317788 (2009f:35071)

[8] H. Berestycki, T. Gallouet and O. Kavian, Nonlinear Euclidean scalar field equations in the plane, C. R. Acad. Sci. Paris Ser. I Math., 297 (1983), 307-310. MR734575 (85e:35041)

[9] H. Berestycki and P.-L. Lions, Nonlinear scalar field equations. I. Existence of a ground state, Arch. Rational Mech. Anal., 82 (1983), 313-345. MR695535 (84h:35054a)

[10] P. Clément And G. Sweers, Existence and multiplicity results for a semilinear elliptic eigenvalue problem, Ann. Scuola Norm. Sup. Pisa Cl. Sci. (4), 14 (1987), 97-121. MR.937538 (89j:35053) 
[11] E.N. Dancer, Some mountain-pass solutions for small diffusion, Differential Integral Equations, 16 (2003), 1013-1024. MR1989600 (2004e:35063)

[12] M. Del Pino and P.L. Felmer, Spike-layered solutions of singularly perturbed elliptic problems in a degenerate setting, Indiana Univ. Math. J., 48 (1999), 883-898. MR.1736974 (2001b:35027)

[13] M. del Pino, P. Felmer and J. Wei, On the role of distance function in some singular perturbation problems, Comm. Partial Differential Equations, 25 (2000), 155-177. MR 1737546 (2000m:35017)

[14] B. Gidas, W. N. Ni and L. Nirenberg, Symmetry and related properties via the maximum principle, Comm. Math. Phys., 68 (1979), 209-243. MR544879 (80h:35043)

[15] R. Gardner and L. A. Peletier, The set of positive solutions of semilinear equations in large balls, Proc. Roy. Soc. Edinburgh Sect. A, 104 (1986), 53-72. MR877892 (88e:35063)

[16] D. Gilbarg and N. S. Trudinger, Elliptic Partial Differential Equations of Second Order. Second edition, Grundlehren Math. Wiss. 224, Springer, Berlin, 1983. MR.737190 (86c:35035)

[17] J. JANG, On spike solutions of singularly perturbed semilinear Dirichlet problems, J. Differential Equations, 114 (1994), 370-395. MR1303033 (95i:35099)

[18] L. Jeanjean and K. TAnakA, A remark on least energy solutions in $R^{N}$, Proc. Amer. Math. Soc., 131 (2003), 2399-2408. MR1974637 (2004c:35127)

[19] P.L. Lions, Symetrie et compacite dans les espaces de Sobolev, J. Funct. Anal., 49 (1982), 315-334. MR683027 (84k:46027)

[20] P.L. Lions, The concentration-compactness principle in the calculus of variations. The locally compact case. II. Ann. Inst. Henri Poincaré, 1 (1984), 223-283. MR.778974 (87e:49035b)

[21] Y.Y. Li And L. NiREnBerG, The Dirichlet problem for singularly perturbed elliptic equations, Comm. Pure Appl. Math., 51 (1998), 1445-1490. MR.1639159 (99g:35014)

[22] W.M. Ni, I. TAKAGi AND J. WEI, On the location and profile of spike-layer solutions to a singularly perturbed semilinear Dirichlet problem: intermediate solutions, Duke Math. J., 94 (1998), 597-618. MR1639546 (99h:35011)

[23] W.M. Ni AND J. WeI, On the location and profile of spike-layer solutions to singularly perturbed semilinear Dirichlet problems, Comm. Pure Appl. Math., 48 (1995), 731-768. MR:1342381 (96g:35077)

[24] E. NOUSSAIR AND S. YAN, The effect of the domain geometry in singular perturbation problems, Proc. London Math. Soc., 76 (1998), 427-452. MR.1490244 (98m:35013)

[25] M. H. Protter and H. F. Weinberger, Maximum Principles in Differential Equations, SpringerVerlag, New York, Berlin, Heidelberg and Tokyo, 1984. MR762825 (86f:35034)

[26] M. Struwe, Variational Methods. Application to Nonlinear Partial Differential Equations and Hamiltonian Systems, Springer-Verlag, 1990. MR.1078018 (92b:49002)

[27] J. Wei, On the construction of single-peaked solutions to a singularly perturbed semilinear Dirichlet problem, J. Differential Equations, 129 (1996), 315-333. MR1404386 (97f:35015)

Department of Mathematics, Pohang University of Science and Technology, Pohang, Kyungbuk 790-784, Republic of Korea

E-mail address: jbyeon@postech.ac.kr 